INVESTIGACIÓN

Recibido: 18/06/2020 --- Aceptado: 14/12/2020 --- Publicado: 12/03/2021

\title{
LA RADIOTERAPIA PARA EL TRATAMIENTO DEL CÁNCER EN LA PUBLICIDAD DE PRINCIPIOS DEL S. XX
}

\author{
Radiotherapy for the treatment of cancer in the advertising of the early \\ 20th century
}

(1) 87 R $R^{6}$ Laura Almudéver Campo': Universidad de Valencia. España.

Laura.Almudever@uv.es

(D) $\mathbb{R}^{\circ}$ "enán E. Camaño Puig: Universidad de Valencia. España.

Ramon.Camano@uv.es

\section{RESUMEN}

El descubrimiento de los rayos $\mathrm{X}$ y del radium generó gran curiosidad entre los científicos. Ambos elementos representaban la cura milagrosa que podría tratar una amplia gama de enfermedades mortales, como el cáncer, pudiendo llegar a colmar las expectativas de la población. El objetivo de dicho artículo es analizar las inserciones publicitarias relativas al radium y los rayos $\mathrm{X}$ aplicadas para el tratamiento del cáncer, en la prensa española a comienzos del siglo XX (1903-1912), en los periódicos $A B C$ y La Vanguardia. Para ello, a partir de la hemeroteca digital de ambos medios se seleccionaron todas y cada una de las veces que se pudo identificar el término "cáncer" en los anuncios publicitarios, para posteriormente seleccionar únicamente aquellos cuyo tratamiento ofertado para la cura del cáncer fuera la radioterapia. En total se publicaron un total de 162 anuncios, 146 para $A B C$ y 16 para La Vanguardia. Se puede afirmar que el tratamiento proporcionado por ambos medios es bastante diferente en términos numéricos, aunque en los aspectos cualitativos hay niveles de coincidencias importantes. La publicidad fue sobre todo redaccional, aunque poco a poco la imagen empezó a cobrar protagonismo, los mensajes publicitarios fueron depurándose y mejoró el tratamiento tipográfico, algo que se nota de manera incipiente en nuestro grupo de anuncios. Todo ello nos permite hablar de la progresiva consolidación de un mercado publicitario desde el primer decenio del siglo XX.

PALABRAS CLAVE: Publicidad - Cáncer - tratamiento - rayos X - radioterapia prensa - España.

${ }^{1}$ Laura Almudéver Campo: Doctora en Enfermería y Licenciada en Periodismo por la Universidad de Valencia. Profesora asociada Facultad de Enfermería y Podología de la Universidad de Valencia. 


\begin{abstract}
The discovery of X-rays and radium generated great curiosity among scientists. Both elements represented the miracle cure that could treat a wide range of deadly diseases, such as cancer, and could meet the expectations of the population. The objective of this article is to analyze the advertising inserts related to radium and Xrays applied to cancer treatment, in the Spanish press at the beginning of the 20th century (1903-1912), in the $A B C$ and La Vanguardia newspapers. To do this, each and every one of the times that the term "cancer" could be identified in advertisements was selected from the digital archive of both media, to later select only those whose treatment offered for the cure of cancer was the radiotherapy. A total of 162 ads were published, 146 for $A B C$ and 16 for La Vanguardia. It can be affirmed that the treatment provided by both means is quite different in numerical terms, although in the qualitative aspects there are important levels of coincidences. The advertising was mainly editorial, although little by little the image began to take center stage, the advertising messages were refined and the typographical treatment improved, something that is incipiently noted in our group of ads. All this allows us to speak of the progressive consolidation of an advertising market since the first decade of the 20th century.
\end{abstract}

KEY WORDS: Advertising - Cancer - Treatment - X-rays - Radiotherapy - Press Spain.

\title{
A RADIOTERAPIA PARA O TRATAMENTO DE CÂNCER NA PUBLICIDADE DE COMEÇOS DO S. XX
}

\section{RESUMO}

A descoberta dos raios $\mathrm{X}$ e do radium gerou grande curiosidade entre os cientistas. Ambos elementos representavam a cura milagrosa que poderia tratar uma grande variedade de doenças mortais, como o câncer, sendo capaz de atender as expectativas da população. O objetivo do artigo é analisar as inserções publicitárias relativas ao radium e os raios $\mathrm{X}$ aplicadas para o tratamento do câncer, na imprensa espanhola nos começo do século XX (1903-1912), e nos jornais ABC e La Vanguardia. Para isto, a partir da biblioteca digital de ambos os meios foram escolhidas todas e cada uma das vezes que é identificada a palavra "câncer" nas propagandas, para posteriormente selecionar únicamente aqueles onde o tratamento ofertado para a cura do câncer fosse a radioterapia. No total, publicaram-se um total de 162 propagandas, 146 para $A B C$ e 16 para La Vanguardia. Pode-se afirmar que a abordagem utilizada por ambos os meios é bastante diferente em termos numéricos, embora nos aspectos qualitativos haja níveis de coincidências importantes. A publicidade foi maioritariamente redacional, mesmo que pouco a pouco a imagem começou a ter protagonismo, as mensagens publicitárias foram sendo depuradas e melhorou o tratamento tipográfico, algo que se percebe de forma incipiente no nosso 
grupo de propagandas. Tudo isso permite concluir a progressiva consolidação de um mercado publicitário desde o primeiro decênio do século XX.

PALAVRAS CHAVE: Publicidade - Câncer - tratamento- raios X - radioterapia imprensa - Espanha.

\section{Cómo citar el artículo:}

Almudéver Campo, L. y Camaño Puig, R. E. (2021). La radioterapia para el tratamiento del cáncer en la publicidad de principios del s. XX. Vivat Academia. Revista de Comunicación, 154, 1-21. http://doi.org/10.15178/va.2021.154.e1249 http://www.vivatacademia.net/index.php/vivat/article/view/1249

\section{INTRODUCCIÓN}

Podríamos decir que la historia de los rayos $\mathrm{X}$ comienza con los experimentos del científico británico William Crookes, pero fue el físico Wilhelm Conrad Röntgen quien llevó a cabo los experimentos con los tubos Crookes y la bobina de Ruhmkorff, mediante los que analizó los rayos catódicos que condujeron a la identificación de los rayos X. Procedió a la repetición de varios experimentos y determinó que los rayos creaban una radiación muy penetrante, pero invisible; observando que atravesaban grandes capas de papel e incluso metales menos densos que el plomo.

Röntgen siguió probando hasta descubrir que esos rayos atravesaban no sólo el cartón, sino también la madera e incluso las planchas de aluminio como si fueran transparentes. Como desconocía la naturaleza de esos rayos capaces de atravesarlo casi todo, Röntgen los denominó rayos $\mathrm{X}$, ya que esa era la letra más utilizada como incógnita en ecuaciones matemáticas. El 28 de diciembre de 1895, presentó un escrito, en el que recogía sus observaciones, a la Sociedad Físico-Médica de Würzburg, que se publicó el 6 de enero del año siguiente con el título de Eine neue Art von Strahlen (Sobre una nueva clase de rayos. Comunicación preliminar), por el que recibió docenas de premios y distinciones científicas. De este modo, los rayos $\mathrm{X}$ se convirtieron en un avance científico crucial que revolucionó la práctica médica.

La noticia del descubrimiento se propagó a una asombrosa velocidad, pues para sorpresa del propio Röntgen, apareció publicada el 5 de enero en el Neue Freie Presse, cuyo propietario era el padre de un amigo suyo al que le había mostrado el comunicado y las extraordinarias imágenes. El 7 de enero The Standard daba a conocer el "sensacional descubrimiento" a los ciudadanos de Londres, el 8 lo publicaba el New York Times, el 10 Le Petit Parisien y el 31 de enero de 1986, aparecía publicado en el periódico español La Vanguardia con el titular: "El descubrimiento del Dr. Röntgen. La fotografía a través de los cuerpos opacos" (La Vanguardia, página 4 del 31 enero 1896). 
El descubrimiento de Röntgen generó gran curiosidad entre los científicos. Entre ellos la joven polaca, Marie Sklodowska, que en 1896 se encontraba en París desarrollando su Doctorado en la Escuela de Física, decidió realizar su tesis sobre la "radioactividad espontánea" utilizando diferentes materiales. En el curso de sus investigaciones llegó a la conclusión de que el torio era más radiactivo que el uranio, de lo cual informó a la Academia de Ciencias de París el 12 de abril de 1898. En esa presentación sugiere que la radiactividad es una propiedad atómica, pues es independiente del estado físico o químico del material radiactivo y también predice que se podrían encontrar elementos más activos que los conocidos.

En 1895, habiéndose casado con Pierre Curie, otro físico prominente que trabajaba en la Soborna, ambos desarrollan métodos para investigar nuevos elementos y, en julio de 1898, separaron por dilución de la pechblenda un nuevo elemento que llamaron polonio. En diciembre del mismo año, procedieron a la precipitación del polonio y obtuvieron un nuevo elemento radiactivo, al cual denominan radio. Para producir una muestra de este nuevo elemento recibieron una tonelada de mineral de uranio donada por el gobierno austriaco.

Durante cuatro años el matrimonio trabajó para llegar a obtener finalmente la décima parte de un gramo de radio puro. Ambos, los rayos $\mathrm{X}$ y el radium, en su aplicación provocaron múltiples efectos biológicos que fueron identificados con relativa prontitud, comenzando a aplicar esta radiación como elemento diagnóstico y terapéutico (Cornejo, 2004).

La aplicación médica de los rayos $X$ fue inmediata, primero para localizar cuerpos extraños en los tejidos y diagnosticar fracturas óseas, y posteriormente, tuvo otras aplicaciones (Casas, 1996), entre las que se deben considerar los numerosos experimentos biológicos llevados a cabo durante la primera década del siglo XX para determinar la acción de estas radiaciones sobre los más variados tipos de células, tejidos y organismos, observándose efectos reductores en la división celular, con una mayor radiosensibilidad de los tejidos en crecimiento, y efectos teratogénicos en los embriones (Bohn, 1903; Danysz, 1903).

La angustia frente al cáncer avanzado y los dolores que ocasionaba esta enfermedad incurable motivó, en los primeros tiempos, el uso indiscriminado de la radioterapia, convirtiéndola en un tratamiento empírico, administrado por médicos y cirujanos generales, que se llevaba a cabo en consultorios particulares en vez de centros académicos. El radium se distribuía en forma de sales y se aplicaba en el cuerpo en todas las formas imaginables: placas en tubos, pomada radioactiva, compresas de lino o de yodo radioactivo, inyecciones y suspensión para su ingestión oral; calculando la dosis a administrar según la respuesta al tratamiento.

La observación de que la proximidad o el contacto del radium con la piel provocaba lesiones a modo de quemaduras, similares a las producidas por los rayos X, fueron descritas ya en 1900 por los alemanes Walkhoff (1900) y Giesel (1900), siendo además experimentadas incluso por sus descubridores. Becquerel en 1901 se produjo una quemadura en el vientre por llevar radium en un tubo de ensayo en el

Vivat Academia. Revista de Comunicación. 2021, n 154, 1-21 
bolsillo de su chaleco y Pierre Curie se produjo deliberadamente una reacción similar en su antebrazo, comunicando ambos los efectos de la radiación sobre la piel, en junio de dicho año, a la Academia de Ciencias de París.

De igual modo poco después, en noviembre de 1901, Foveau de Courmelles (1904), tras analizar la reacción cutánea sufrida por él mismo al llevar radium en uno de sus bolsillos, adjudicó a este elemento propiedades biológicas que definió como "químicas, penetrantes y destructivas". De ahí surgió la idea de su utilización en el tratamiento de determinados procesos dermatológicos. Por ello el matrimonio Curie prestó un tubo de radium a Henri Danlos, dermatólogo del Hospital de St. Louis de París, para su experimentación, siendo el primer médico en tratar con radium a un paciente afecto de lupus (Danlos y Bloch, 1901).

Así pues, en los primeros años del siglo XX, las indicaciones de la radiumterapia apuntaban más a afecciones cutáneas no malignas (acné, eczema, lupus eritematoso, psoriasis, hipertricosis, nevus, etc.), aunque también se experimentó en algunos tipos de cáncer cutáneo no infiltrante. Esto no impidió que su posible efecto destructivo, se terminara aplicando también en tumores cutáneos infiltrantes y de otras estructuras corporales como el cérvix, el útero o el recto, con eficacia meramente paliativa y efectos secundarios tales como quemaduras y necrosis de tejidos sanos.

Knight (1986) planteó que los científicos sabían que la radiación de los rayos X y el radio era similar, pero el radio era considerado la versión natural de los rayos $\mathrm{X}$ e indicaba que, en esos momentos, en todo el mundo, la gente creía que el radio era maravilloso y que poseía propiedades medicinales. Se decía que disminuía el estreñimiento y la presión arterial, curaba el insomnio aliviando los nervios, aumentaba la actividad sexual y se ponía en todo tipo de productos, desde cremas para la piel a pastas dentales; todo sobre los nuevos rayos fue deslumbrante y fascinante para el público, provocando una manía de rayos $\mathrm{X}$ generalizada que siguió al anuncio del descubrimiento de los rayos $\mathrm{X}$, la sorprendente "nueva luz".

Por otra parte, debe indicarse que el radium, en esta época se promocionó en base al efecto beneficioso que se atribuía a la radiactividad ante numerosas patologías. Los rayos $\mathrm{X}$ representaban la cura milagrosa que podría curar una amplia gama de enfermedades mortales, pudiendo llegar a colmar las expectativas de la gente en este sentido. Se podría decir que fue un momento de desconcierto aprovechado por charlatanes, carentes de toda base científica.

El descubrimiento de los rayos $\mathrm{X}$, tal y como hemos visto (apareció en los medios antes de su comunicado oficial), estaba constantemente en los periódicos, proporcionando ideas de evidencia y eficacia de la ciencia. No es sorprendente que este tipo de procesos vinculados a los rayos $X$ tuviera un atractivo interesante desde la perspectiva del marketing, incluso para productos que no tenían nada que ver con ciencia o medicamentos. Los anunciantes podían contar con un efecto publicitario añadido, lo que convirtió los rayos X, en 1900, en una fuerza invisible máxima, todo 
ello antes incluso de que se pudiera pensar en ningún tipo de regulación sobre bienes de consumo o protección de los consumidores.

De hecho, el primer anuncio publicitario en el que se utilizó este descubrimiento incorporó la radiografía obtenida, por Röntgen, de la mano de su esposa, y apareció en la revista Electrical Engineer, solo cuatro meses después del anuncio del descubrimiento de Röntgen, insertado por una empresa de Thomas Alva Edison (Seliger, 1995). Por tanto, no es sorprendente que se hayan utilizado los rayos X para fines de diagnóstico y posiblemente para terapia a las pocas semanas del anuncio del descubrimiento, dada la fascinación casi ubicua del público y su inmediata aplicación generalizada en la medicina.

\section{OBJETIVOS}

El objetivo de este trabajo es analizar las características de las inserciones publicitarias relativas al radium y los rayos $\mathrm{X}$ realizadas en los medios de comunicación escrita de nuestro país, en una sociedad de bajo nivel de alfabetización. Este estudio, por tanto, pretende analizar la publicidad relativa a los productos y servicios que se ofertaban a principios del siglo XX en relación con productos como el radium y los rayos $\mathrm{X}$ en relación con una enfermedad como el cáncer, realizando en lo posible, una aproximación a la realidad social y consideraciones que se tenía acerca de estos productos en el periodo de tiempo comprendido entre el año 1903 y 1912; situándolos en términos contextuales y determinando la imagen que se transmitía junto con el comportamiento publicitario y alcance de las palabras, conceptos, ideas o imágenes empleadas a través de la publicidad insertada en los medios de comunicación escritos.

En definitiva, tratar de conocer la imagen transmitida a través de la publicidad sobre estos productos y su impacto en cuestiones relativas al cáncer, concretamente en los dos diarios de información general y de mayor difusión en el ámbito geográfico del Estado Español, de ideología aparentemente distinta, el $A B C$, publicado en Madrid, y La Vanguardia, publicado en Barcelona, así como analizar y comparar los resultados obtenidos.

\section{METODOLOGÍA}

Todo trabajo de investigación supone un primer paso de análisis de la literatura existente sobre la temática elegida, así como aquellos aspectos que se acercan a la investigación propuesta (la selección, el análisis, el estudio de los documentos y las publicaciones). A partir de aquí, establecimos una serie de criterios para la selección de las publicaciones analizadas que se han basado en la elección de dos diarios de información general de mayor difusión en el ámbito geográfico del Estado Español, desde el 1 de enero de 1903 hasta el 31 de diciembre de 1912. Los diarios que han cumplido este criterio, y que han pasado a integrar la muestra de este proyecto, son los periódicos $A B C$ y La Vanguardia, publicados uno Madrid y otro en Barcelona, respectivamente. Adicionalmente y en base a esta característica se han obtenido dos 
ejemplos representativos de las dos ciudades españolas con mayor población en los años de nuestro estudio, con lo que conforman una parte esencial del nuevo periodismo español.

Los dos periódicos analizados han sido consultados en su versión digital a través de su hemeroteca digital. Se realizó la búsqueda de los anuncios publicitarios que incluyeran el concepto cáncer. Posteriormente se realizó una lectura de los mismos para seleccionar únicamente aquellos cuyo tratamiento ofertado para la cura del cáncer fuera la radioterapia.

\section{RESULTADOS}

El número de periódicos analizados incluidos en el estudio fue de 7.044, en concreto 3.414 periódicos de $A B C$ de Madrid junto con su suplemento Blanco y Negro, y 3.630 periódicos de La Vanguardia.

Una vez obtenida la muestra de estudio, se seleccionaron todos los anuncios publicitarios en los que aparecía el concepto cáncer, tal y como se muestra en la Tabla 1., en la cual se encuentra el número anuncios por periódico, año y sus sumatorios.

Tabla 1. Anuncios publicitarios con el concepto cáncer.

\begin{tabular}{|l|c|c|c|c|c|c|c|c|c|c|c|}
\hline Descriptor/Año & 1903 & 1904 & 1905 & 1906 & 1907 & 1908 & 1909 & 1910 & 1911 & 1912 & TOTAL \\
\hline Publicidad ABC & --- & --- & 12 & 21 & 22 & 19 & 26 & 73 & 68 & 38 & $\mathbf{2 7 9}$ \\
\hline Publicidad LV & 40 & 1 & 8 & 2 & --- & 8 & 31 & 3 & 3 & 11 & $\mathbf{1 0 7}$ \\
\hline Total & $\mathbf{4 0}$ & $\mathbf{1}$ & $\mathbf{2 0}$ & $\mathbf{2 3}$ & $\mathbf{2 2}$ & $\mathbf{2 7}$ & $\mathbf{5 7}$ & $\mathbf{7 6}$ & $\mathbf{7 1}$ & $\mathbf{4 9}$ & $\mathbf{3 8 6}$ \\
\hline
\end{tabular}

Fuente: Elaboración propia.

Cuantitativamente, en el año 1910 el periódico $A B C$ fue el que mayor número de anuncios relativos al cáncer publicó, con un total de 73 (26\%3) unidades de análisis, seguido del año 1911 con 68 (24'3\%) anuncios encontrados y de 1912, con 38 (13'8\%) anuncios. Por otra parte, en el periódico La Vanguardia, el año en el que se publicaron mayor número de anuncios fue 1903, con un total de 40 (37²\%) unidades de análisis, seguido de 1909, con 31 anuncios y un $29 \%$.

En la siguiente Tabla 2. Anuncios publicitarios con el concepto cáncer que ofertaban curación mediante radioterapia, se puede apreciar que el número de anuncios publicados en $A B C$ respecto a este ámbito fue de 146, y en el caso de $L a$ Vanguardia, el número de anuncios fue de 16.

Tabla 2. Anuncios publicitarios con el concepto cáncer que ofertaban curación mediante radioterapia.

\begin{tabular}{|l|l|l|l|l|l|l|l|l|l|l|l|}
\hline Descriptor/Año & 1903 & 1904 & 1905 & 1906 & 1907 & 1908 & 1909 & 1910 & 1911 & 1912 & TOTAL \\
\hline
\end{tabular}


Almudéver Campo, L. y Camaño Puig, R. E.

La radioterapia para el tratamiento del cáncer en la publicidad de principios del s. XX

\begin{tabular}{|l|c|c|c|c|c|c|c|c|c|c|c|}
\hline Publicidad ABC & --- & --- & 2 & 2 & 1 & & 7 & 61 & 58 & 15 & $\mathbf{1 4 6}$ \\
\hline Publicidad LV & & & & & & & 13 & 1 & 1 & 1 & $\mathbf{1 6}$ \\
\hline Total publicidad & & & 2 & 2 & 1 & & 20 & 63 & 59 & 16 & $\mathbf{1 6 2}$ \\
\hline
\end{tabular}

Fuente: Elaboración propia.

En total se publicaron un total de 162 anuncios que pasamos a describir a continuación, para lo que procederemos a ordenar los anuncios en base al medio y la fecha de publicación, comenzando por el periódico $A B C$.

El primer anuncio que encontramos tiene unas características singulares, se trata de un anuncio que aparece publicado en diez ocasiones que no se rige por ningún patrón especial de publicación, pero aparece dos veces cada año: 1905, 1909, 1910, 1911, 1912. Se inserta con la apariencia de noticia, pero en realidad es publicidad. Es enviado desde París (Francia), por una "correspondencia especial" y habla de la curación, casi milagrosa de enfermedades -entre ellas el cáncer- llevadas a cabo por el profesor G. A. Mann, que "causan la más viva curiosidad, gran asombro y no menos admiración". Pretende haber descubierto una cierta Ley de la Naturaleza que aparentemente describe en el libro que promociona, en entre otras cosas, en el anuncio.

En dos formatos distintos, uno para el periódico $A B C$ y otro para La Vanguardia, cabe resaltar el titular y subtítulos, que decían así: "Con su poder fenomenal, este hombre opera milagros". "Los ciegos ven y los paralíticos andan. Los enfermos desahuciados por los médicos son curados por él. No existe enfermedad que no pueda ser curada. Hace desaparecer los dolores, cierra heridas, cura el cáncer, la tuberculosis, los tumores, y opera maravillas que asombran a la Medicina moderna y desafían toda explicación". Asimismo, ofrece consultas gratuitas y cura a los enfermos en sus casas, sin verlos, tan fácilmente como si estuvieran en su presencia, afirmando que a todo aquel que quiera escribirle, le indicará las causas de los síntomas que le aquejan y la manera de obtener la curación por la radiopatía. Se trata de una publicidad de grandes dimensiones en comparación con otros anuncios de la que se desprende mucha esperanza e ilusión para los enfermos de cáncer.

El siguiente anuncio aparece publicado dos veces en 1906, concretamente el 27 de enero y 3 de febrero. Ocupa toda la página y se trata de un texto muy curioso con un gran titular y muchos subtítulos. En él aparece un huecograbado con la imagen del autodenominado un "Gran Taumaturgo", escrito en negrita y con mayúsculas, que "devuelve la salud a los incurables con el empleo de una fuerza sutil". El contenido del anuncio es similar al anterior, utilizando múltiples testimonios de supuestos pacientes que se habían beneficiado de su tratamiento.

Vivat Academia. Revista de Comunicación. 2021, n 154, 1-21 


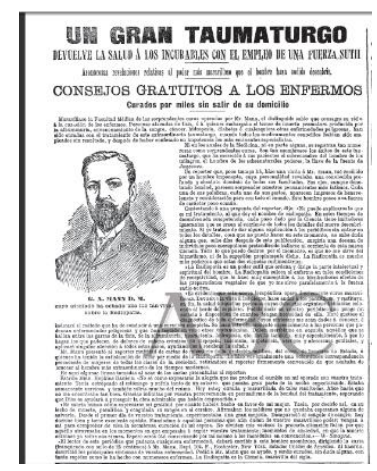

Foto 1: Anuncio publicitario Un gran taumaturgo.

Fuente: Periódico ABC (27 de enero de 1906, página 2).

El siguiente anuncio solo aparece publicado el día 6 de abril de 1907, y se anuncia que el Dr. Díaz de la Quintana puede tratar diversas patologías, entre ellas, el cáncer, con Baños de luz, Electricidad y Rayos X, con una primera visita al precio de 5 pesetas.

Un nuevo anuncio titulado "El radio y los tumores malignos" aparece publicado cuatro veces entre octubre y noviembre de 1909. Se ofrece una consulta "completamente gratuita" para difundir los beneficios de la terapia con radium, destacando la apertura de un establecimiento de la Sociedad Española del Radio y sus Aplicaciones con la mira humanitaria de difundir su conocimiento y extender sus beneficios.

El 26 de noviembre de 1909 apareció publicado una única vez el siguiente anuncio, con un titular en negrita "Instituto del Radio". Indicando la disponibilidad de "Los últimos progresos" para tratamientos de enfermedades de la piel y mucosas, mediante la utilización de tratamientos sencillos y sin el más leve dolor con consultas matinales de diez a doce, al precio de siete pesetas y de cinco y media a seis y media, gratuita.

Se siguió publicitando el Instituto del Radio con nuevos anuncios, como el que sigue a continuación, que aparece seis veces de enero a abril de 1910. Cambia el formato y se destaca en el titular el concepto del RADIO en negrita y su uso en dolencias incurables hasta el momento. En el texto informa de la cura de enfermedades mortales como el cáncer a través de las sales de radio, que con sus "inagotables radiaciones transforman las lesiones sin dejar huellas apreciables y sin el más leve dolor". Dichos tratamientos se realizan en el Instituto del Radio, único en España, en horario de mañanas (de pago) y de tardes (gratuito). Además, se indica que se puede ir a presenciar los tratamientos para cerciorase de la comodidad de éstos y ver así, los resultados.

En el siguiente anuncio, aparece por primera vez publicitado el Instituto Radiumterapéutico en fecha de 25 de febrero de 1910, aunque no se anuncie tal cual. Se informa que el Doctor D. A. Gallego Cepeda ha adquirido los aparatos del radium 
para curar diferentes patologías, entre ellas el cáncer e indicando las características del radium así como el "enorme precio de tan codiciado metal. Un solo gramo cuesta cuatrocientos mil francos".

$\mathrm{Al}$ siguiente mes se publicita de forma masiva un anuncio, que se repite setenta veces de marzo de 1910 a diciembre del 1912, con una publicación semanal durante largos periodos de tiempo. El titular Instituto Radiumterápico de Madrid se encuentra en el Paseo de Recoletos, 31. Por medio del radium se curan, sin operaciones quirúrgicas, grandes molestias, como el cáncer, lupus, angiomas, etc., que hasta el momento no desaparecían con ninguno de los recursos empleados.

Asimismo, en 1910 aparece publicitado tres veces dicho establecimiento, aunque llama la atención la fotografía de un paciente de 59 años con un epitelioma ulcerado, y una fotografía posterior tras ser tratado con radio, indicando la bonanza del tratamiento a través de la imagen.

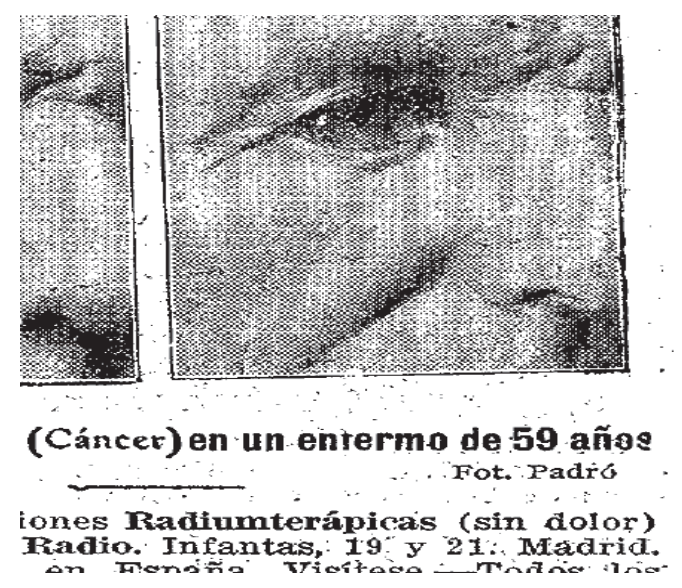

Foto 2: Anuncio publicitario Epitelioma ulcerado en un enfermo de 59 años.

Fuente: Periódico $A B C$ (17 de septiembre de 1910, página 20).

El siguiente anuncio fue publicado seis veces entre marzo y abril de 1910. Se informa del poder de la Radiumterapia con "informes gratis" y "tratamientos a domicilio" tal y como se indica al final del anuncio. Tal y como dice el anuncio: "Todo sin dolor y sin dejar huellas. El summum de la comodidad y la estética". Indica que las consultas se llevaran a cabo en el único Instituto del Radio establecido en España con todos los elementos radiumterápicos, en Madrid. Calle Infantas, 19 y aunque aporta un horario, indica que las clínicas están abiertas desde las 9 de la mañana a las 7 de la noche con informe gratis y tratamiento a domicilio.

El siguiente anuncio apareció cinco veces publicado entre mayo y junio de 1910. Lo que llama la atención es la innovadora introducción de la fotografía de la fachada donde se encuentra ubicado el Instituto Radiumterápico de Madrid. Es igual que un anuncio anterior, con texto idéntico, aunque añaden que es una "lástima que estos beneficios extraordinarios no puedan ser utilizados más fácilmente por el enorme precio de tan escaso y codiciado metal". 


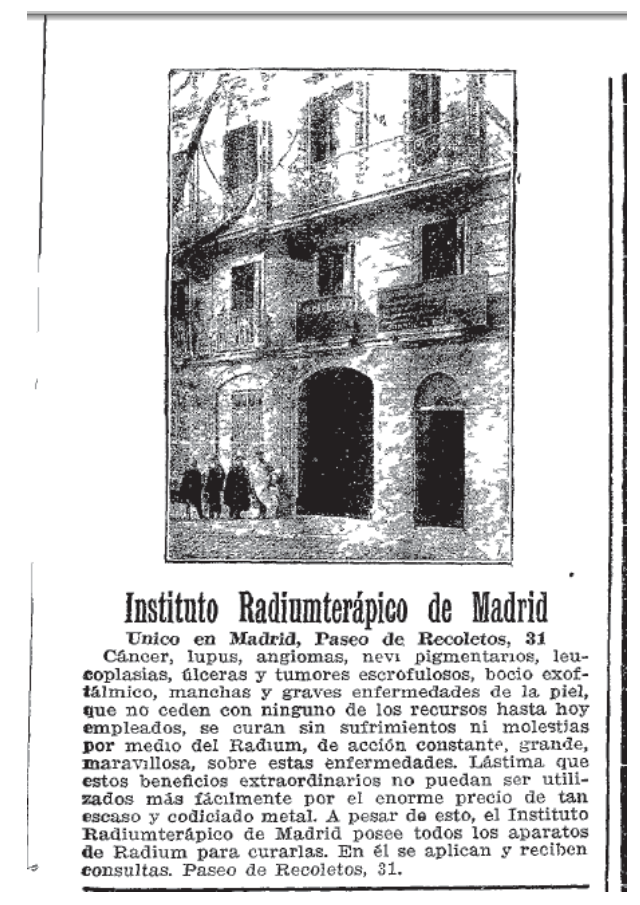

Foto 3: Anuncio publicitario Instituto Radiumterápico de Madrid.

Fuente: Periódico $A B C$ (15 de mayo de 1910, página 20).

El siguiente anuncio apareció publicado tres veces en junio de 1910. Se destaca el mensaje que se quiere transmitir en la última frase: “En él se aplican y reciben consultas sin los enormes gastos para los enfermos que generalmente exigen las intervenciones quirúrgicas." Se trata del mismo texto que otros anuncios, con alguna variación en el texto, y adicionalmente se indica que un solo gramo de radium cuesta 400.000 francos.

Un nuevo anuncio del Instituto del Radio comentaba la característica de "Primera fundación en España", apareciendo seis veces entre junio, julio y agosto de 1910. Estaba ubicado en la calle Infantas, 19 y 21 y aportó como novedad un número de teléfono, indicando las horas de visita y el precio de ésta. Se trata de una variación de otro anuncio, casi idéntico.

Como continuación al anuncio anterior, encontramos un nuevo anuncio del Instituto del Radio, que apareció seis veces entre agosto y noviembre de 1910, añadiendo la foto de la fachada del Instituto del Radio, cuyo rótulo aparece sobre la puerta de entrada y través de la cual se informa de la institución que se trata. Posiblemente, la introducción de la fotografía sea resultado de la oferta por parte del periódico, o también, podría estar condicionada por la introducción de la fotografía de la fachada, por parte de sus competidores del Instituto Radiumterápico, en su publicidad. Como texto incorpora nuevamente la característica de "Primera fundación en España", dirección, número de teléfono, indicando las horas de visita y precio de ésta. 


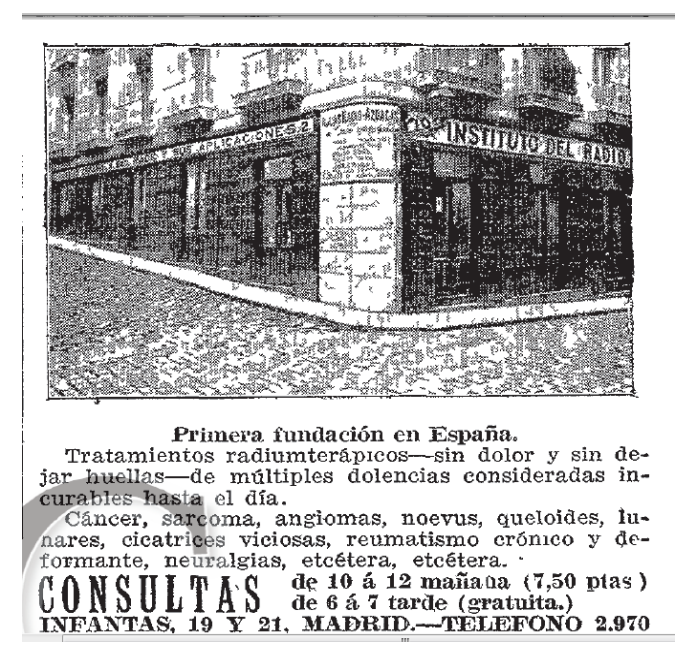

Foto 4: Anuncio publicitario Instituto del Radio.

Fuente: Periódico ABC (28 de agosto de 1910, página 20).

El Instituto Radiumterápico de Madrid, incluye tres veces de octubre a noviembre de 1910 un nuevo anuncio simplificado, informando de las enfermedades que pueden ser curadas mediante el Radium, únicamente en sus instalaciones.

Otras cuatro veces aparece un nuevo anuncio del Instituto Radiumterápico de Madrid, entre enero y febrero de 1911, en el que se describen todas las afecciones que pueden ser tratadas mediante el Radium. En ellos se establece una analogía con establecimientos similares de París y Londres e informa de la dirección del establecimiento.

El siguiente anuncio solo aparece publicado en una ocasión, concretamente el 27 de noviembre de 1910 en la página 20. También se hace una comparativa entre el antes y después de un cáncer ulcerado e infiltrado en una paciente de cincuenta años curada con aplicaciones radiumterápicas sin dolor. Ofertando la posibilidad de ser visitados para obtener informes y antecedentes, con testimonios múltiples. Todos los días de 6 a 7 de la tarde e inspección médica gratuita.

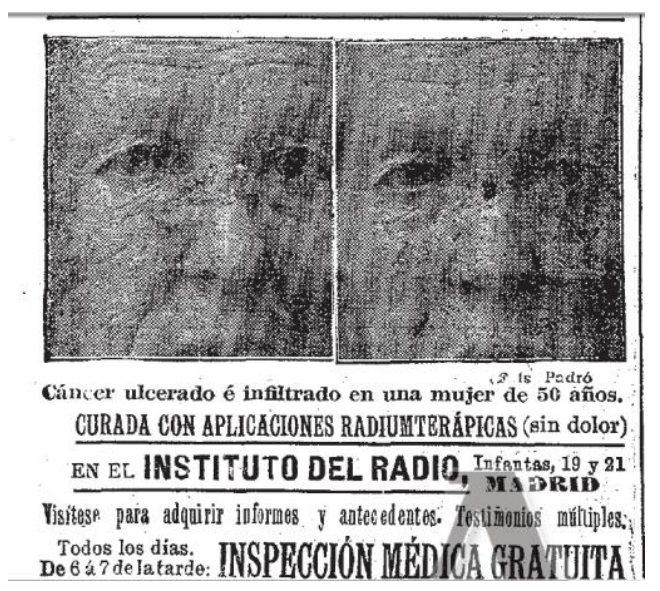


Foto 5: Anuncio publicitario Cáncer ulcerado e infiltrado en una mujer de 50 años.

Fuente: Periódico $A B C$ (27 de noviembre de 1910, página 20).

Un nuevo anuncio aparece publicado trece veces entre noviembre de 1911 y diciembre de 1912, bajo el epígrafe de "Publicidad Recomendada". Se trata del Instituto Radiumterapéutico de Madrid, en donde se plantea que es "constante en los que padecen enfermedades conocidas con el nombre de cáncer, ..., oírlos manifestar que están cansados de emplear inútilmente varios procedimientos para curarse y verlos sufrir hasta su muerte desesperada por dolores y molestias". Indicando que los que se encuentran en este caso no se han sometido al tratamiento por el Radium, en el Instituto Radiumterápico de Madrid. En el Instituto Radiumterápico “hubiesen observado desde las primeras aplicaciones, todos, sensible mejoría, y la mayor parte de ellos, su curación".

Un nuevo anuncio apareció publicado el 6 de mayo de 1912, en la página 12, bajo la sección de Publicidad Recomendada y el titular "Emanatorio del Radium". En este anuncio después de realizar la enumeración de diferentes médicos y clínicas se plantea que se ha establecido el emanatorio para la curación de enfermedades internas. A continuación enumera algunas de la enfermedades de aplicación: el reumatismo, gota, artritis deformantes en todas sus formas y variedades $y$ complicaciones; anquilosis, enfermedades del corazón y riñones, afecciones de los huesos y de las articulaciones, restos de exudados en las membranas serosas (pleuresías, peritonitis) enfermedades del sistema nervioso (epilepsia, histerismo, neuralgias, paresias, parálisis, hemiplejías, tabes dorsal y neurastenia) afecciones de naturaleza escrofulosa, infartos viscerales y graves enfermedades de la piel, son tan notables, que parecen providenciales por los rápidos beneficios que reportan al paciente. Informando de lo inofensivo de la emanación y de su absorción a nivel pulmonar e indicando que excita determinadas funciones orgánicas como la hematopoyesis, páncreas, la actividad del hígado, tiroides y otros órganos. Consulta, de 3 a 5 en el Paseo de Recoletos al objeto de que los enfermos de Madrid puedan disfrutar de tan poderoso medio de curación

En la La Vanguardia el número de anuncios es más reducido, comenzando por uno que apareció doce veces entre marzo y agosto de 1909. Indicando que en la "clínica del Dr. Torres se cura el cáncer mediante fulguración por rayos X", y se informa que tal doctor es exalumno del Hospital de París y su consulta está ubicada al parecer en la Plaza Beato Oriol de la ciudad de Barcelona.

El siguiente anuncio solo fue publicado el día 3 de marzo de 1910, en la página 3. Coincide con otro anuncio del periódico $A B C$, donde se publicita el Instituto Radiumterapéutico, nombrando al doctor Gallego Cepeda y al igual que en éstos, indica las diferentes patologías para las que se va a utilizar el radium, haciendo una loa de las características del radium y el "enorme precio de tan codiciado metal. Un solo gramo cuesta cuatrocientos mil francos", indicando que a pesar de esto, Gallego Cepeda después de estudiar y conocer perfectamente la técnica de su aplicación ha 
comprado los correspondientes aparatos y plantea la dirección en Madrid en la que se aplican y recibe las consultas.

Por último, aparece publicado tres veces: el 23 de octubre de 1910, el 19 de noviembre de 1911 y el 15 de diciembre de 1912, un anuncio del Profesor Mann que inserta el mismo anuncio incorporado previamente en el periódico $A B C$, de Madrid, con ligeras modificaciones tipográficas.

Tal y como puede verse en la Tabla 3. Distribución de los anunciantes por medio, año y características de los anuncios, que incluimos a modo de compilación, podríamos decir que se trataba de un mercado reducido y más o menos delimitado, solo competían dos entidades, fundamentalmente, en la ciudad de Madrid, aunque dos profesionales parece que quisieron abrirse espacio en el mercado en 1097 y 1909, los doctores Díaz y Torres, uno en Madrid y otro en Barcelona. Aunque el máximo de la actividad se desarrollaba en Madrid con un alto nivel de competencia entre el Instituto del Radio y el Instituto de Radiumterapia, este último llegó a anunciarse en La Vanguardia, para atraer a los habitantes de Barcelona a recibir tratamiento en sus instalaciones.

Tabla 3. Distribución de los anunciantes por medio, año y características de los anuncios

\begin{tabular}{|l|l|l|l|l|l|l|l|}
\hline \multicolumn{2}{|l|}{ Anunciante } & Medio & \multicolumn{2}{l|}{ Fecha ler anuncio } & \multicolumn{2}{l|}{ Características anuncio } \\
\hline 1 & Prof. G. Mann & ABC & 1905 & Enero & Texto & \\
\hline 2 & Prof. G. Mann & ABC & 1906 & Enero & Texto & Huecograbado \\
\hline 3 & Dr. Díaz de la Quintana & ABC & 1907 & Abril & Texto & \\
\hline 4 & Dr. Torres Carreras & LV & 1909 & Marzo & Texto & \\
\hline 5 & Instituto del Radio & ABC & & Octubre & Texto & \\
\hline 6 & Instituto del Radio & ABC & & Noviembre & Texto & \\
\hline 7 & Instituto del Radio & ABC & 1910 & Enero & Texto & \\
\hline 8 & $\begin{array}{l}\text { Instituto } \\
\text { Radiumterapia }\end{array}$ & de & & Febrero & Texto & \\
\hline 9 & $\begin{array}{l}\text { Instituto } \\
\text { Radiumterapia }\end{array}$ de & LV & & Marzo & Texto & \\
\hline 10 & $\begin{array}{l}\text { Instituto } \\
\text { Radiumterapia }\end{array}$ & ABC & & Marzo & Texto & \\
\hline 11 & $\begin{array}{l}\text { Instituto } \\
\text { Radiumterapia }\end{array}$ & ABC & & Marzo & Texto & $\begin{array}{l}\text { Imagen } \\
\text { masculino }\end{array}$ \\
\hline 12 & Instituto de Radio & ABC & & Marzo & Texto & \\
\hline 13 & $\begin{array}{l}\text { Instituto } \\
\text { Radiumterapia }\end{array}$ & ABC & & Mayo & Texto & Imagen fachada \\
\hline 14 & $\begin{array}{l}\text { Instituto } \\
\text { Radiumterapia }\end{array}$ & ABC & & Junio & Texto & \\
\hline 15 & Instituto de Radio & ABC & & Julio & Texto & \\
\hline 16 & Instituto de Radio & ABC & & Agosto & Texto & Imagen fachada \\
\hline 17 & $\begin{array}{l}\text { Instituto } \\
\text { Radiumterapia }\end{array}$ & de & ABC & & Octubre & Texto & \\
\hline 18 & Prof. G. Mann & LV & & Octubre & Texto & \\
\hline 19 & Instituto & & Noviembre & Texto & Imagen \\
\hline
\end{tabular}


Almudéver Campo, L. y Camaño Puig, R. E.

La radioterapia para el tratamiento del cáncer en la publicidad de principios del s. XX

\begin{tabular}{|l|l|l|l|l|l|l|}
\hline & Radiumterapia & & & & & femenino \\
\hline 20 & $\begin{array}{l}\text { Instituto } \\
\text { Radiumterapia }\end{array}$ & ABC & 1911 & Enero & Texto & \\
\hline 21 & Instituto de Radio de & ABC & 1911 & Noviembre & Texto & \\
\hline 22 & $\begin{array}{l}\text { Instituto } \\
\text { Radiumterapia }\end{array}$ & ABC & 1912 & Mayo & Texto & \\
\hline
\end{tabular}

Fuente: Elaboración propia.

Al mismo tiempo es remarcable la utilización de la imagen que realiza el Instituto de Radiumterapia, que lleva a cabo dos anuncios con la utilización de los resultados de su tratamiento en personas y una imagen de la fachada de su establecimiento. El instituto del Radio lleva a cabo un anuncio de lo que fueron sus primeros pasos, La Sociedad Española del Radio, posteriormente constituido como Instituto del Radio. El instituto de Radiumterapia, llevó a cabo 10 anuncios de los 22 del periodo estudiado. Finalmente, un dato que es necesario destacar es que se ha encontrado el grueso de los anuncios concentrado, 13 anuncios, en el año 1910.

\section{DISCUSIÓN}

Uno de los aspectos más relevantes en esos años fue el descubrimiento y aplicación del radium para la curación del cáncer, tal y como informaban los periódicos. En 1906, doce libros y más de 80 artículos ya habían aparecido sobre radioterapia en la literatura médica norteamericana, en los que se mencionaba sus ventajas, destacando que es indolora, deja pequeñas cicatrices sin desfigurar, destruye el tejido enfermo, alivia el dolor y reduce el miedo a una operación. Lo cierto es que la nueva modalidad originó un gran entusiasmo por su aplicación. Este interés supuso la creación del Laboratorio Biológico del Radium en París en 1906, el "Radiumhemmet" de Estocolmo en 1910, el Instituto del Radium en Londres en 1911, y la Sociedad Norteamericana del Radium en 1916.

Ya en 1903, concretamente el 29 de julio en la página 4, La Vanguardia decía que Mr. Mackenziw Davison acababa de tratar con éxito un caso de cáncer por medio del radium.

Meses después, el 24 de noviembre de 1903, en la página10, $A B C$ informaba sobre La Real Sociedad de Londres, que había entregado una medalla a M. Curie, por haber descubierto las propiedades del radium contra el cáncer. Al año siguiente, en la página 6 del 23 de enero de 1904, el doctor Morton ocupaba una noticia en el $A B C$, sobre la influencia satisfactoria del radio en el cáncer desarrollado sobre la piel. Y en la portada del 30 de abril de 1904, La Vanguardia se hacía eco de una noticia que anunciaba la milagrosa terapia para el cáncer con la fosforescencia terapéutica. También se informaba sobre los efectos curativos del radio y de la luz en los atacados del cáncer, los días 11 de julio y 29 de octubre de 1904 en la página 4 de La Vanguardia. 
Y es que parece ser que el progreso hacía maravillas y que la aplicación de radium debía considerarse prodigiosa, al menos, así se anunciaba La Vanguardia en la página 5 , del 5 de mayo de 1905. Al año siguiente, en 1906, se publicaban cuatro noticias en referencia a la aplicación del radium en la terapéutica del cáncer, definiendo el cáncer como uno de los grandes azotes incurables, junto con la tuberculosis. Asimismo, se afirmaba que los rayos Röntgen no podían producir el cáncer ni transformar una úlcera simple en una úlcera cancerosa (19 de agosto de 1906 en La Vanguardia, página 7).

Desde 1906 hasta 1911, no se informaba sobre el radium en ninguno de los dos periódicos analizados. Es el 16 de abril de 1911, en la página 11, cuando $A B C$ informa sobre el empleo del radium en la curación del cáncer por parte de D. Alfredo Gallego Cepeda. Y en la página 3 del 13 de junio de 1912, La Vanguardia notificaba sobre el uso de bromuro de radium, que puesto en una placa de cuatro centímetros y mediante filtros de papel de estaño, había curado un cáncer de párpado y borde de la órbita ocular. Sin embargo, las informaciones sobre el radium no se centraban solamente en noticias, sino también en anuncios publicitarios. A lo largo de nuestro análisis, hemos encontrado una gran cantidad de publicidades que versaban sobre el tratamiento del cáncer, las aquí consideradas han sido en referencia al radium o los rayos $X$.

En lo relativo a la publicidad del Profesor Mann, es necesario tratarla aquí, aunque no está vinculada con el radium o los Rayos X, fundamentalmente porque es la muestra de que todo nuevo ámbito científico, o de negocio, genera habitualmente un grupo de embaucadores y charlatanes que utilizando una semántica similar intentan utilizar los ambientes favorables para sacar partido. En este caso, una tecnología completamente nueva que podría ver a través del tejido humano vivo, que incluso prometía ser la primera respuesta no quirúrgica efectiva al cáncer, en donde la imagen de la radiografía transmitía una sensación de tecnología punta y a la vez parecía magia, una fuerza invisible que podría haber estado operando siempre. La magia ha estado en el corazón de la religión y la religión es un sistema paralelo a la publicidad (Gerson, 2004:547).

El público en general consideró que los rayos $\mathrm{X}$, pronto podrían usarse de manera rutinaria para todo, desde el diagnóstico y tratamiento más simple al más complejo. Los rayos representaban la cura milagrosa que algún día, con solo pulsar un interruptor, podría curar una amplia gama de enfermedades mortales. Gerson recoge en su escrito la opinión de otros autores y expone que uno de ellos planteaba que el campo de la radiología en esos momentos era como un "verdadero país de las hadas de la ciencia" en el que las esperanzas más extravagantes algún día podrían realizarse (p. 546). Los rayos $\mathrm{X}$ y el radium atraparon la imaginación de los científicos y el público, este último, simplemente quedó asombrado con los rayos $\mathrm{X}, \mathrm{y}$ los charlatanes como el profesor Mann jugaron con esta fascinación al realizar sutiles referencias en su discurso a la nueva tecnología. 
De hecho, si buscamos en el diccionario la palabra Radiopatía, que en palabras de Mann decía era la "Ciencia, maravilla del siglo", encontramos que se trata de una enfermedad provocada por radiación, en especial, la producida por la manipulación de los rayos $\mathrm{X}$ y del radio. Bajo el nombre de radiopatía o radiolesión se agrupan todos los trastornos relacionados o producidos por los rayos $\mathrm{X}$ y los cuerpos radioactivos como quemaduras, heridas que podrían transformarse en cáncer, ulceraciones sanguíneas, trastornos oculares, etc. Por tanto, podemos inferir que el Profesor Mann, un individuo bien formado y conocedor de ciertos avances, que se inviste de autoridad denominándose a sí mismo 'Profesor' y que oferta curas milagrosas con el empleo de una fuerza sutil, comienza a utilizar esta palabra una vez se hace, en muchos casos evidente en los medios científicos, los problemas, radiopatías o lesiones provocadas por la utilización de los rayos $\mathrm{X}$ o el radium sin considerar hacer referencia a las mismas.

Adicionalmente, aparecieron siete tipos diferentes de publicidades para anunciar los servicios que ofrecía el Instituto del Radio ubicado en la calle Infantas 19 y 21 de Madrid. En todos se insertan grandes titulares, en los que se informa de los nuevos tratamientos por el radio aplicados a múltiples dolencias incurables hasta ese momento. Dicho instituto se anuncia como uno de los pioneros en aplicar los últimos progresos de la medicina, siendo el único establecimiento y la primera fundación en España. En total, 30 veces han aparecido estos anuncios en sus siete formatos distintos, todos ellos en el periódico $A B C$.

Otro de los anuncios que también se ha publicado con asiduidad con respecto al radium trata sobre el Instituto Radiumterápico de Madrid, ubicado en Paseo de Recoletos, 31. Se ha publicado 104 veces en diez formatos diferentes. Todos ellos, han sido publicados en $A B C$, a excepción de uno, que lo hizo en La Vanguardia. Tras el estudio de estos anuncios se desprende la gran utilidad que va adquiriendo la publicidad que se plasma en la famosa frase de "quien no se anuncia no se vende" (Rodríguez, 2007), lo que se traduce en que existían establecimientos de los cuales no se tenían noticias porque no se anunciaban, demostrando que la publicidad en el periodo que estamos estudiando servía y mucho, para sus propósitos.

Los anuncios publicitarios, reclamaban a los posibles compradores con muy pocas armas: las tipográficas y el recurso del verso o cantar las excelencias del producto. Poco a poco los comerciantes se van dando cuenta de los efectos que los anuncios producen en los posibles consumidores y como consecuencia, la publicidad va adquiriendo un peso importante en los periódicos. Los anuncios publicitarios habían sido hasta ese momento, en su mayoría, textos que se insertaron en las columnas verticales, utilizando tipografías diferentes para destacar los productos publicitados, y a veces incluyeron ilustraciones muy simples, copiadas de publicaciones extranjeras (Rodríguez, 2007).

Durante los primeros años del siglo, fue sobre todo publicidad redaccional, en la que las ilustraciones eran casi anecdóticas, pero a medida que avanzamos en el tiempo la imagen llegó a protagonizar casi en exclusiva el anuncio en detrimento del

Vivat Academia. Revista de Comunicación. 2021, nº 154, 1-21 
texto, que, a partir de entonces, acompaña a la imagen. Algo que se nota de manera incipiente en nuestro grupo de anuncios, donde encontramos en el caso de profesor Mann, una imagen de su persona en huecograbado. En los siguientes anuncios en donde se inserta una imagen aparecen fotografías de la fachada de los centros de tratamiento y, en dos casos, se utiliza la imagen de pacientes por parte del Instituto del Radio para mostrar sus logros. Obviamente esto sería impensable en estos momentos, pero la carencia de regulación, en el momento, lo hizo posible.

Los anuncios mostraron cada vez en mayor medida a consumidores disfrutando de los beneficios del uso de determinados artículos, y así la publicidad comenzó a mostrar los beneficios del consumo, a vender sensaciones y a enseñar las vidas de los consumidores mejoradas por el uso de los productos ofertados. Fue a mediados de la primera década del siglo XX, cuando la publicidad fue progresivamente asentándose en los diarios y revistas, ocupando cada vez mayor espacio en ellos, y dejando atrás la maquetación de todos los anuncios en páginas fijas para ir distribuyéndose a lo largo de toda la publicación.

En estos primeros años del siglo XX, el mercado publicitario en prensa se caracterizó por su inestabilidad. La escasez de anunciantes y la poca diversidad de los productos publicitados eran el reflejo de que en aquellos años los primeros pasos de la sociedad de consumo en España eran todavía muy incipientes. La pobre presentación gráfica de los anuncios, salvo algunos casos aislados y excepcionales, nos informa del precario y todavía poco desarrollado mercado publicitario, pero en el que se percibían dos procesos diferentes, por una parte el desarrollo del mercado publicitario que pasa del texto a una utilización más frecuente de la imagen, pasando de huecograbado a fotografía y por otra, la diseminación de información sobre avances tecnológicos, especialmente y en este caso para la curación de problemas de salud, en donde los profesionales, una vez vislumbran las posibilidades de negocio, y a la vez de cierta competencia, utilizan la publicidad para ofrecer sus servicios.

\section{CONCLUSIONES}

A lo largo de nuestro trabajo hemos analizado la publicidad en relación con la aplicación del radio para el tratamiento del cáncer en dos diarios de ideología, aparentemente distinta, el $A B C$ y $L a$ Vanguardia. El tratamiento proporcionado por ambos medios es bastante diferente en términos numéricos, aunque en los aspectos cualitativos hay niveles de coincidencias importantes. La inserción de publicidad es uno de los puntos en los que se da de manera más asidua, publicándose en ambos medios anuncios idénticos de manera total o parcial.

En nuestro caso, la publicidad es determinante pero también podría decirse esto de una manera más minoritaria acerca de los aspectos preventivos, dada la ideología predominante que identificaba el cáncer como un proceso infeccioso. Las informaciones acerca del cáncer publicadas en la prensa de más circulación en España en la horquilla temporal que comprende de 1903 a 1912, corresponden preferentemente al ámbito publicitario en donde se ofertaban tratamientos

Vivat Academia. Revista de Comunicación. 2021, n 154, 1-21 
innovadores, en unos años, en los que la enfermedad del cáncer era aún muy desconocida. Este marcado sesgo en la información sobre el cáncer pudo propiciar, en la sociedad, una visión excesivamente optimista del tratamiento del cáncer, de sus resultados y de su pronóstico, pues se ofertaban tratamientos que decían ser "milagrosos" y poseían propiedades curativas "excelentes" para la curación total del cáncer.

Hemos visto, en conclusión, que, en los primeros años del siglo XX en España, la prensa aumentó el número de páginas dedicadas a la publicidad, al mismo tiempo que se estabilizó su ubicación en la misma y se incrementó el número de anunciantes y de productos publicitados. Mejoró el tratamiento tipográfico de los anuncios, la imagen fue adquiriendo un creciente protagonismo y los mensajes publicitarios fueron depurándose. Estos datos nos permiten hablar de la progresiva consolidación de un mercado publicitario desde el primer decenio del siglo XX.

Así pues, la publicidad fue el escaparate privilegiado a través del cual se presentaron los nuevos productos que comenzaron a inundar el mercado, a la vez que se constituyó como la herramienta por excelencia para introducir nuevas pautas y hábitos de consumo en la sociedad, sirviendo además como anticipadora y difusora de los nuevos estilos de vida, de las nuevas costumbres y de los nuevos roles de la sociedad de consumo. Aunque hay que pensar también, dado lo visto, que la publicidad puede ser también utilizada por charlatanes y falsarios para llevar a cabo actividades fraudulentas.

Existe un importante elemento a considerar en este apartado, el acceso a la lectura, dado que es determinante para poder decodificar las informaciones contenidas en los periódicos de la época. Es necesario tenerlo en cuenta puesto que un porcentaje muy alto de la población no sabía leer ni escribir, lo que determinaba el acceso al conocimiento preventivo y terapéutico respecto de problemas de salud, tales como el cáncer. Todo ello, independientemente de que las teorías predominantes en el ámbito científico de la actuación oncológica fueran más o menos acertadas.

La pertenencia a un nivel social u otro establecía el nivel de alfabetización y, por tanto, era concluyente para poder acceder a las fuentes de información respecto a la salud, una situación que hoy como ayer se mantiene. El cáncer es una enfermedad con un gran impacto en nuestra sociedad, y la percepción que tenga un individuo del riesgo de padecerlo o de las acciones que pudieran ejercerse para prevenirlo o incluso de la utilización de los servicios sanitarios depende entre otros factores, de la información recibida a través de los medios de comunicación.

\section{REFERENCIAS}

Becquerel, H. y Curie, P. (1901). L'action physiologique des rayons du radium. Cr Acad Sci Paris, 132, 1289-91. 
Bohn, G. (1903). L'influence du radium sur les animaux en voie de croissance. $\mathrm{Cr}$ Acad Sci Paris, 136, 1012-3.

Casas, F. (1996). De la röntgenlogia a l'oncologia radioteràpica: contribució a cent anys de lluita contra el càncer a Catalunya. 1886-1996. Barcelona: Prasfarma.

Cornejo, L. M. (2004). El descubrimiento del radium y la radioterapia. Historia de la radioterapia en Panamá. Revista Médico Científica, 17(1). http://www.revistamedicocientifica.org/index.php/rmc/article/view/55

Danlos, M. y Bloch, P. (1901). Note sur le traitement du lupus erithèmateux Par des applications de radium. Ann Dermat Syphil, 2, 986-8 26.

Danysz, J. (1903). De l'accion pathogéne des rayons et emanation du radium sur les différents tissus et différents organismes. Cr Acad Sci Paris, 137, 1296-8.

Foveau de Courmelles, F. (1904). Les applications medicales du radium. Paris: Farjas.

Gerson, E. S. (2004). Scenes from the Past. X-ray Mania: The X Ray in Advertising, Circa 1895. RadioGraphics, 24(2), 544-551. https://pubs.rsna.org/doi/pdf/10.1148/rg.242035157

Giesel, F. (1990). Ueber radioactive Stoffe. Berichte Dtsch Chem Gesell, 33, 3569-71.

Knight, N. (1986). The new light: x-rays and medical futurism. In: Corn J, ed. Imagining tomorrow: history, technology, and the American future. Cambridge, Mass: MIT Press.

Rodríguez Martín, N. (2007). Anunciar es vender: Nacimiento y desarrollo de la publicidad en la España del primer tercio del siglo XX. Prensas Universitarias de Zaragoza.

Seliger, H. H. (1995). Wilhem Conrad Röntgen and the glimmer of light. Physics today, 48(11), 25-3.

Walkhoff, F. O. (1900). Unsichtbare, photographisch wirksame Strahlen. Photographische Rundschau, 14, 189-91.

\section{AUTORES:}

\section{Laura Almudéver Campo}

Doctora en Enfermería por la Universidad de Valencia (2016), posee el máster de Enfermería Oncológica (2011), es Licenciada en Periodismo (2007) y Diplomada en Enfermería (2003). Ha desempañado su labor como enfermera asistencial en diferentes hospitales y centros de salud. 
Compagina su labor asistencial con la docencia, impartiendo clase como profesora asociada en la Facultad de Enfermería de la Universidad de Valencia. Asimismo, desde el 2007 forma parte de la redacción de la revista Enfermería Integral, del Colegio Oficial de Enfermería de Valencia, siendo coordinadora de la misma desde 2015. Laura.Almudever@uv.es

Orcid ID: https:// orcid.org/0000-0002-3049-4879

Google Scholar: https://scholar.google.com/citations?hl=es\&user=8ZVj28cAAAAI

Research Gate: https://www.researchgate.net/profile/Laura-Almudever-Campo

Scopus: https:// www.scopus.com/authid/detail.uri?authorId=57216888854

\section{Ramón E. Camaño Puig}

Obtuvo el título de Doctor Europeo (1997), por Leeds Metropolitan University (UK), es enfermero y Licenciado en Comunicación Audiovisual por la Universidad de Valencia. Actualmente, es profesor Titular en la Universidad de Valencia, ha participado en diferentes programas de la Unión Europea y publicado diferentes artículos y libros sobre temas de comunicación y salud.

Ramon.Camano@uv.es

Orcid ID: https:// orcid.org/0000-0002-8018-2232

Google Scholar: https://scholar.google.com/citations?hl=es\&user=oIZ0w7oAAAAI

Research Gate: https://www.researchgate.net/profile/Ramon_Camano-Puig

Scopus: https://www.scopus.com/authid/detail.uri?authorId=25821697300 